\title{
Neurological outcomes after hematopoietic stem cell transplantation for cerebral X-linked adrenoleukodystrophy, late onset metachromatic leukodystrophy and Hurler syndrome
}

Desfechos neurológicos após transplante de células tronco hematopoiéticas na adrenoleucodistrofia ligada ao X, forma cerebral, na leucodistrofia metacromática de início tardio e na síndrome de Hurler

Jonas Alex Morales Saute1,2,5, Carolina Fischinger Moura de Souza', Fabiano de Oliveira Poswar 7 , Karina Carvalho Donis $s^{1,3}$, Lillian Gonçalves Campos 4,5 , Adriana Vanessa Santini Deyl ${ }^{6}$, Maira Graeff Burin', Carmen Regla Vargas ${ }^{1,13}$, Ursula da Silveira Matte ${ }^{7,8,9}$, Roberto Giugliani, 1,3,5,7,8,9, Maria Luiza Saraiva-Pereira ${ }^{1,2,7,10,}$ Leonardo Modesti Vedolin ${ }^{4,5}$, Lauro José Gregianin ${ }^{6,12}$, Laura Bannach Jardim 1,2,3,5,7,11

\begin{abstract}
Hematopoietic stem cell transplantation (HSCT) is the only available treatment for the neurological involvement of disorders such as late-onset metachromatic leukodystrophy (MLD), mucopolysaccharidosis type I-Hurler (MPS-IH), and X-linked cerebral adrenoleukodystrophy (CALD). Objective: To describe survival and neurological outcomes after HSCT for these disorders. Methods: Seven CALD, 2 MLD and 2 MPS-IH patients underwent HSCT between 2007 and 2014. Neurological examinations, magnetic resonance imaging, molecular and biochemical studies were obtained at baseline and repeated when appropriated. Results: Favorable outcomes were obtained with 4/5 related and 3/6 unrelated donors. Two patients died from procedure-related complications. Nine transplanted patients were alive after a median of 3.7 years: neurological stabilization was obtained in 5/6 CALD, 1/2 MLD, and one MPS-IH patient. Brain lesions of the MPS-IH patient were reduced four years after HSCT. Conclusion: Good outcomes were obtained when HSCT was performed before adulthood, early in the clinical course, and/or from a related donor.
\end{abstract}

Keywords: hematopoietic stem cell transplantation; leukodystrophy, metachromatic; mucopolysaccharidosis I; adrenoleukodystrophy.

\footnotetext{
${ }^{1}$ Hospital de Clínicas de Porto Alegre, Serviço de Genética Médica, Porto Alegre RS, Brasil;

${ }^{2}$ Hospital de Clínicas de Porto Alegre, Laboratório de Identificação Genética, Porto Alegre RS, Brasil;

${ }^{3}$ Universidade Federal do Rio Grande do Sul, Programa de Pós-Graduação em Saúde da Criança e do Adolescente, Porto Alegre RS, Brasil;

${ }^{4}$ Hospital de Clínicas de Porto Alegre, Serviço de Radiologia, Porto Alegre RS, Brasil;

${ }^{5}$ Universidade Federal do Rio Grande do Sul, Programa de Pós-Graduação em Ciências Médicas, Porto Alegre RS, Brasil;

${ }^{6}$ Hospital de Clínicas de Porto Alegre, Serviço de Oncologia Pediátrica, Porto Alegre, Brasil;

־Universidade Federal do Rio Grande do Sul, Programa de Pós-Graduação em Genética e Biologia Molecular; Porto Alegre RS, Brasil;

${ }^{8}$ Hospital de Clínicas de Porto Alegre, Laboratório de Terapia Gênica, Porto Alegre RS, Brasil;

'Universidade Federal do Rio Grande do Sul, Departamento de Genética e Biologia Molecular, Porto Alegre RS, Brasil;

${ }^{10}$ Universidade Federal do Rio Grande do Sul, Departamento de Bioquímica, Porto Alegre RS, Brasil;

${ }^{11}$ Universidade Federal do Rio Grande do Sul, Departamento de Medicina Interna, Porto Alegre RS, Brasil;

${ }^{12}$ Universidade Federal do Rio Grande do Sul, Departamento de Pediatria, Porto Alegre RS, Brasil;

${ }^{13}$ Universidade Federal do Rio Grande do Sul, Faculdade de Farmacia, Porto Alegre, Brasil
}

Correspondence: Laura Bannach Jardim; Serviço de Genética Médica do Hospital de Clinicas de Porto Alegre; Rua Ramiro Barcelos, 2350; 90035 -903 Porto Alegre RS, Brasil; Email: ljardim@hcpa.edu.br

Conflict of interest: There is no conflict of interest to declare.

Support: C.F.M. de Souza received travel grants and speaker honoraria from Genzyme, which has products for MPS-I treatment. R. Giugliani is an investigator in clinical trials sponsored by Genzyme and Shire, and has received travel grants and speaker honoraria from these companies, which have products for MPS-I and MLD treatment, respectively.

C.R.Vargas, U.S. Matte, R. Giugliani, M.L. Saraiva-Pereira and L.B. Jardim received research fellowships from Conselho Nacional de Desenvolvimento Científico e Tecnológico (CNPq), Brazil.

Received 17 June 2016 ; Received in final form 01 August 2016 ; Accepted 24 August 2016. 
O transplante de células tronco hematopoiéticas (TCTH) é o único tratamento disponível para o envolvimento neurológico de doenças como a leucodistrofia metacromática (MLD), a mucopolissacaridose tipo I-Hurler (MPS-IH) e a adrenoleucodistrofia (CALD). Objetivos: Descrever a sobrevida e os desfechos neurológicos após o TCTH nessas doenças. Métodos: Sete pacientes CALD, 2 MLD e 2 MPS-IH realizaram TCTH entre 2007 e 2014. Avaliações neurológicas, ressonância nuclear magnética e estudos bioquímicos e moleculares foram feitos no baseline e repetidos quando apropriado. Resultados: Desfechos favoráveis foram obtidos em 4/5 TCTH de doadores relacionados e em 3/6 não relacionados. Dois pacientes faleceram de complicações do procedimento. Nove transplantados sobreviveram após uma mediana de 3,7 anos: estabilização neurológica foi obtida em 5/6 CALD, 1/2 MLD e em um caso MPS-IH. As lesões encefálicas de um caso MPS-IH reduziram-se quatro anos após o TCTH. Conclusão: Bons desfechos foram obtidos quando o TCTH foi feito antes da vida adulta, cedo no curso clínico e/ou a partir de um doador relacionado.

Palavras-chave: transplante de células-tronco hematopoiéticas; leucodistrofia metacromática; mucopolissacaridose l; adrenoleucodistrofia

Allogeneic hematopoietic stem cell transplantation (HSCT) was proposed as a treatment for inherited leukoencephalopathies in the eighties, based on the assumption that transplanted macrophages migrate through the blood-brain barrier, and differentiate into microglia that would replace or support native microglia with the functional enzyme through cross-correction ${ }^{1}$. Since then, HSCT has been the only available treatment for the neurological involvement of some lysosomal storage disorders that affect the central nervous system (CNS), such as juvenile and adult metachromatic leukodystrophy (MLD), mucopolysaccharidosis type I Hurler (MPS-IH), and others. In addition, HSCT has been mostly indicated for the cerebral form (CALD) of the peroxisomal disorder X-linked adrenoleukodystrophy (X-ALD) $)^{2,3,4,5,6,7,8,9,10}$. Considering that transplant-related mortality has declined to $10 \%$ and the rate of engraftment has substantially improved in recent years ${ }^{11,12}$, risk of transplant is worthwhile in contrast to the certainty that the natural history of these disorders will lead to dementia, decorticate or vegetative states and death, some years after onset.

There is consensus about the efficacy of HSCT when performed early in life for MPS- $\mathrm{IH}^{9,13,14}$ and at early stages in $\mathrm{CALD}^{6,7,12,15}$. Case reports suggested that HSCT might reduce demyelination in juvenile (onset between two and 14 years) and adult forms of MLD when performed as early as possible $e^{3,4,5,16}$. A recent case control study confirmed that HSCT, at a presymptomatic or early symptomatic stage of juvenile MLD, is associated with disease stabilization ${ }^{10}$.

The aim of the present study was to describe a seven-year experience of a university hospital with HSCT for these disorders, focusing on survival and on neurological outcomes.

\section{METHODS}

\section{Patients and study procedures}

This is a retrospective study of a population consisting of seven CALD, one juvenile and one adult MLD and two MPS-IH patients, who underwent HSCT at Hospital de Clínicas de Porto Alegre between 2007 and 2014. All procedures were covered by the Brazilian Unified Health System, and received the same level of health insurance. Three CALD patients have been reported previously ${ }^{17}$.
Biochemical diagnosis was obtained for all patients and was confirmed by molecular analysis of the appropriate gene. Neurological examinations, neuropsychological tests, magnetic resonance imaging (MRI), and specific biochemical markers were performed before HSCT, and six months to seven years after the procedure.

An HSCT was offered as early as possible when an HLA-matched donor or cord blood was available. Additional clinical criteria for offering HSCT were applied, according to each specific disorder. International recommendations were followed for CALD patients: the presence of white matter lesions in the CNS and a Loes score lower than 10 points? An HSCT was also offered as early as possible following diagnosis of juvenile or adult forms of MLD, provided that some gross motor functions such as walking (with or without aid) were still present. Finally, HSCT was indicated for MPS-IH patients under 2.5 years of age if the clinical status, especially pulmonary function, allowed the procedure ${ }^{9}$.

\section{Biochemical and molecular analyses}

Plasma docosanoic (C22:0), tetracosanoic (C24:0) and hexacosanoic (C26:0) acids were obtained from CALD patients, and analyzed as described previously ${ }^{18}$. Arylsulfatase A (ARSA) activity in leukocyte and a thin-layer chromatography of urinary sulfatides were studied in the MLD patients. In the MPS-IH patients, urinary glycosaminoglycans (GAGs), colorimetric assay (DMB test) and electrophoresis were performed, and $\alpha$-L-iduronidase (IDUA) activity in leukocytes was measured. All biochemical studies were performed at the time of diagnosis: their values were subsequently compared to those obtained after HSCTs.

Molecular analyses of IDUA, ABCDI and ARSA were performed in DNA isolated from peripheral blood prior to the HSCT. In all patients, individual exons and flanking regions amplified by PCR were submitted for Sanger sequencing. For $A R S A$ analysis we also evaluated regions associated with pseudodeficiency (PD) variants using a similar approach ${ }^{19}$.

\section{Brain MRI}

Brain MRI data were obtained with a 1.0 or $1.5 \mathrm{~T}$ systems equipped with a standard circularly polarized head coil. 
Axial fluid-attenuated inversion recovery (FLAIR), axial and coronal $\mathrm{T} 2$ and sagittal $\mathrm{T} 1$ weighted images were obtained in all patients. The MRI was performed at baseline and in the follow-up visits after HSCT. Disease-related MRI scores ${ }^{20,21}$ were reviewed for each patient at the time of the present report. Images were analyzed by two independent neuroradiologists (LMV and LC) blind at the time of the given study. In case of discordant scores, both neuroradiologists conferred together to find a consensus. Any $0.5 \mathrm{~T}$ or low-quality MRI images were excluded from the analysis. An independent researcher (JAMS) rebuilt the time frame later.

\section{HSCT procedures}

The HSCT was performed according to institutional protocol recommendations, and all patients received the myeloablative conditioning regimen consisting of an combination of busulfan and cyclophosphamide, as previously published $^{22}$. Details of the HSCT procedures are described in Table 1.

Ethics, consent and permissions. Consent to publish.

Consents for the transplant procedure and for follow up visits and ancillary tests were obtained from all individuals or their representatives at the time of the procedure, and according to ethical requirements of our institution. This report summarizes results from studies registered as GPPG 13-0390 (for X-ALD), 07-599 (for MLD) and 03-066 (for MPS-IH), all approved by the Ethics Committee (EC) of Hospital de Clínicas de Porto Alegre, following the World Medical Association International Code of Medical Ethics (Declaration of Helsinki).

\section{RESULTS}

The HSCT were performed in 11 patients (six with unrelated donors), in the last seven years. Patients and donor characteristics, conditioning, graft source and engraftment, are shown in Table 1.

Baseline results of the neurological findings, intelligence quotient (IQ), MRI and biochemical markers, as well as the follow-up findings after HSCT, are presented in Table 2. Two patients (one with CALD and one with MPS-IH) died from HSCT complications. Seven of the remaining nine individuals had stabilization of symptoms one year or more following HSCT.

Seven CALD patients underwent HSCT; preliminary data on three of them were reported previously ${ }^{20}$. Case reports are presented in the Supplemental Material. In summary, HSCTs were done in five CALD patient younger than 12 years, in one who was 19.4 years old and in one who was 28.2 years old. The oldest of them also presented with the highest Loes score at the time of his HSCT ( 8 points): he died nine months after the procedure. Five out of the six
CALD patients who survived HSCT were clinically stable at the time of this report (in one patient, the Loes score had even improved). The progression of Loes scores of all seven individuals is shown in Figure 1.

Two MPS-IH underwent HSCT: their data are depicted in Tables 1 and 2, and also on Supplemental Material. Of note, a clear improvement of MRI findings of MPS IH-10 case appeared three years after her HSCT, with significant reduction of periventricular and deep WM lesions. The mild ventricular enlargement also improved (Figure 2).

Due to data scarcity on HSCT efficacy in MLD, individual case reports will be reported bellow.

\section{Patient MLD-8b}

Patient MLD-8b, female, eight years old, was the youngest sister of MLD-8a, the male index patient. A gait disturbance started when MLD-8a was nine years old. He was brought for evaluation at 11 years old: there was loss of cognitive abilities, spastic tetraparesis (wheelchair-bound) and dysarthria. MRIs showed diffuse leukodystrophy and brain atrophy (Eichler score of 21) (Figures 3 A, B, C). ARSA activity in leukocytes was $0.6 \mathrm{nmol} / \mathrm{h} / \mathrm{mg}$ prot (normal range: 5-20) and thin-layer chromatography detected increased sulfatides in urine. ARSA sequencing revealed a homozygosis p.P426L mutation. The family and staff team agreed to not perform HSCT. At the time of the genetic counseling of this family, MLD-8b was eight years old, her school performance was normal and, on clinical examination, only an intermittent strabismus (exotropia) was noted. ARSA activity, urinary sulfatides and $A R S A$ analysis confirmed the diagnosis of MLD. Her Eichler score on MRI was 6 (Figure 3D, Table 2). Patient MLD-8b was transplanted when she was nine years, two months old; her oldest sister did not carry p.P426L mutation at $A R S A$ and was the donor. MRI lesions worsened at the time of HSCT (Eichler score of 10, Figure 3E), but she remained asymptomatic. She started with complex partial seizures at 12 years old, thee years after HSCT. An EEG showed left temporal paroxysmal spikes. Seizures were well controlled with valproic acid. The MRI lesions stabilized until the last observation, five years after HSCT (Eichler score of 12, Figures 3F-G). Her last followup was at 16 years of age: MLD-8b showed normal performance at school and normal neurological examination. Neurophysiological studies depicted subclinical abnormalities (Table 2).

\section{Patient MLD-9}

Patient MLD-9, male, presented with personality changes and cognitive decline at 19 years of age. An MRI scan revealed a diffuse leukodystrophy (Eichler score of 20). No ARSA activity was detectable in his leukocytes and was below normal values in his only brother. He was referred for evaluation almost a year after diagnosis, in order to search for unrelated donors. His family was then studied: 


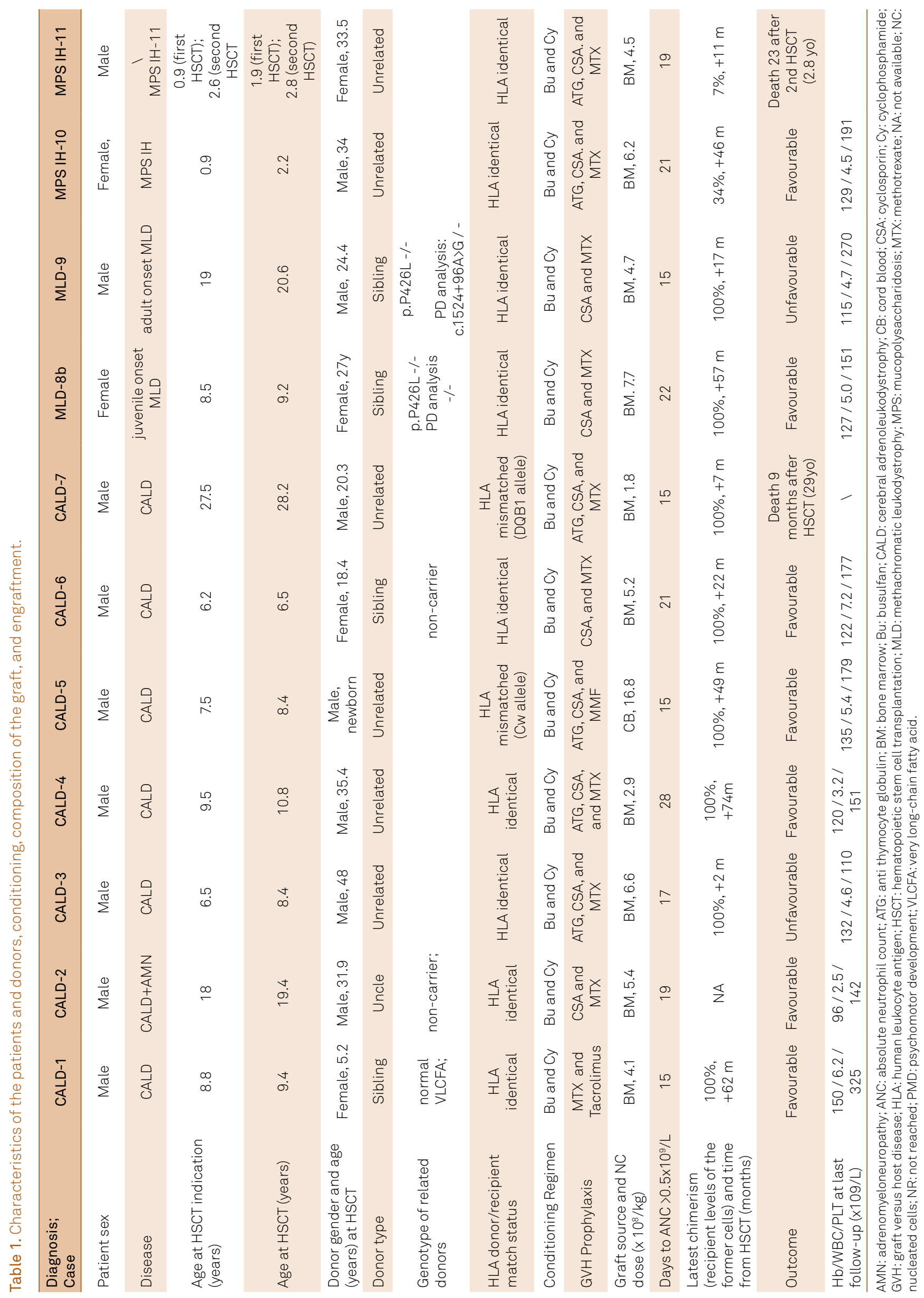




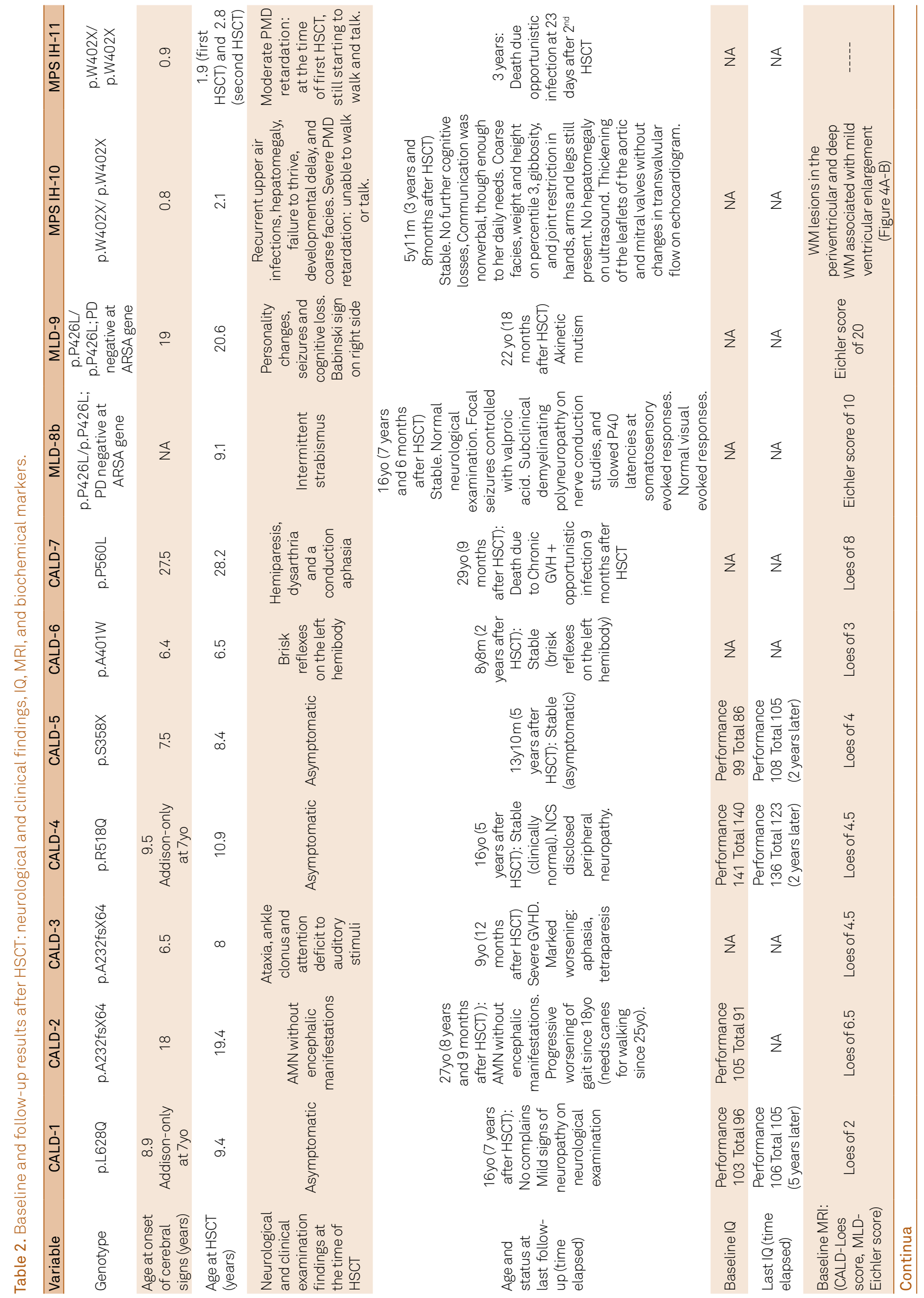




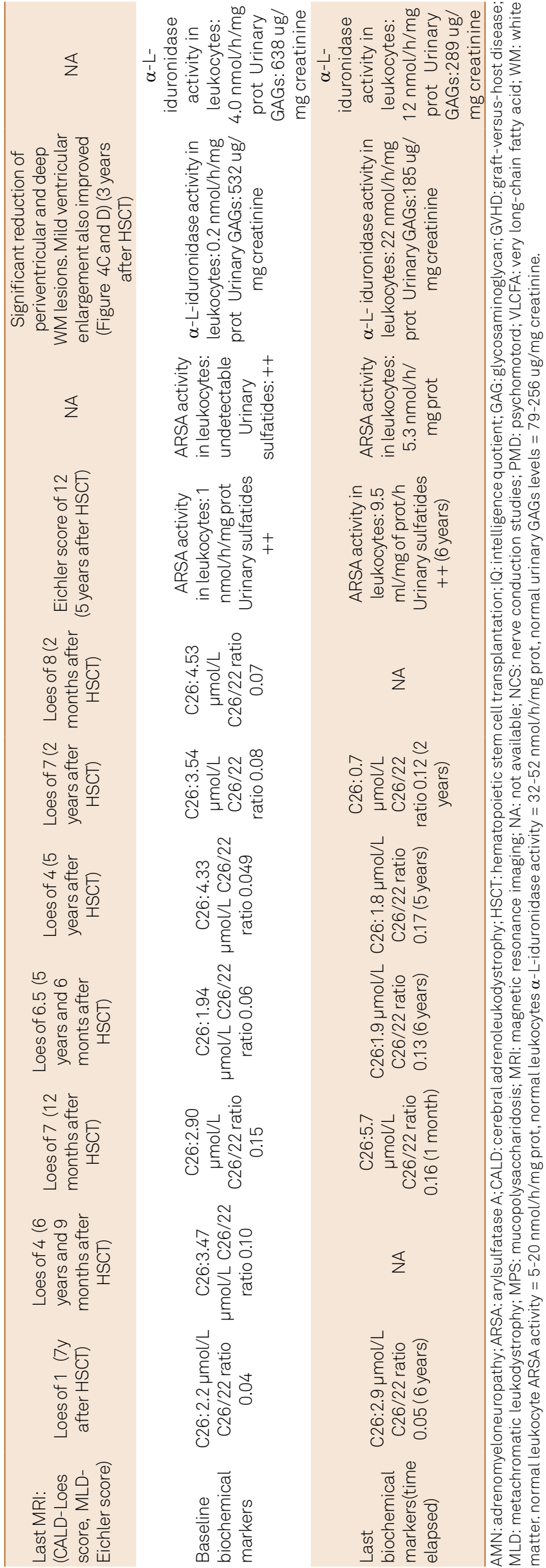

he appears as individual V-14 in Figure 4. Born from consanguineous parents (IV-11 and IV-12, Figure 4), MLD-9 was genotyped as p.P426L/p.P426L; PD - / - at ARSA (pseudodeficiency variants not present). Mother was p.P426L/ - ; $P D$ - / -, and presented with normal ARSA activity and absent urinary sulfatides. Father was p.P426L/-; PD c.1524+96A>G/ -: urinary sulfatides were absent, and ARSA activity was compatible with pseudodeficiency (Figures 3 and 4). The older brother of MLD-9, individual V-13 in Figure 4, was HLA identical to the patient, but was formerly excluded as a candidate donor due to low ARSA activity. He was genotyped as p.P426L - / -; $P D$ - c.1524+96A > G / -: absent urinary sulfatides, and ARSA activity were compatible with pseudodeficiency. He was chosen to be the HSCT donor, performed when the patient was 20 years, seven months old. Patient MLD-9 was discharged 79 days after HSCT. He presented with acute graft-versus-host disease (GVHD) with skin and conjunctival involvement and no other major complications. At the follow-up six months after HSCT, MLD-9 presented with akinetic mutism, being able to walk when forced to, and daily vomiting associated with cyclosporine use. In the last follow-up, 18 months after HSCT (22 years of age), the clinical picture had not changed. His family did not agree to perform MRI and nerve conduction follow-ups.

\section{DISCUSSION}

In the present study, we reported the neurologic progression of Brazilian patients with CALD, MLD, and MPS-IH following HSCT.

These diseases are associated with untreatable and progressive neurological impairment, leading to vegetative states and death ${ }^{11,17,23,24}$. Pathophysiology varies according to gene defect, and notable variability in age at onset and progression rate occurs in all them. To date, HSCT is a unique therapy with potential to bring long-term survival and neurological stabilization for CALD and for some lysosomal storage disorders with brain involvement. Around 500 children with MPS-IH, 114 with MLD, and 465 with CALD underwent HSCT worldwide before $2012^{11,12}$. In the same period, South American HSCT procedures were described in seven CALD patients (the follow-ups of three of them were reported by Jardim et al. ${ }^{17}$ ), six MPS and one Gaucher disease type $1^{25}$. Our present report adds information about four new and three already-reported CALD patients, two new MLD, and two new MPS-IH patients, focusing on their neurological outcomes.

Nine out of our 11 patients (81\%) were alive after a median of 3.7 years after procedure: 6/7 CALD, 2/2 MLD and 1/2 MPS-IH. According to the literature, survival rates improved from $55-65 \%$ in early series ${ }^{6,8,25,26}$ to $85-95 \%$ in the most recent series ${ }^{7,11}$. Our rates were comparable to the latter. A 28-year-old CALD man (CALD-7), and a three-year-old MPSIH boy (MPS-IH-11) died 23 and 270 days, respectively, after 


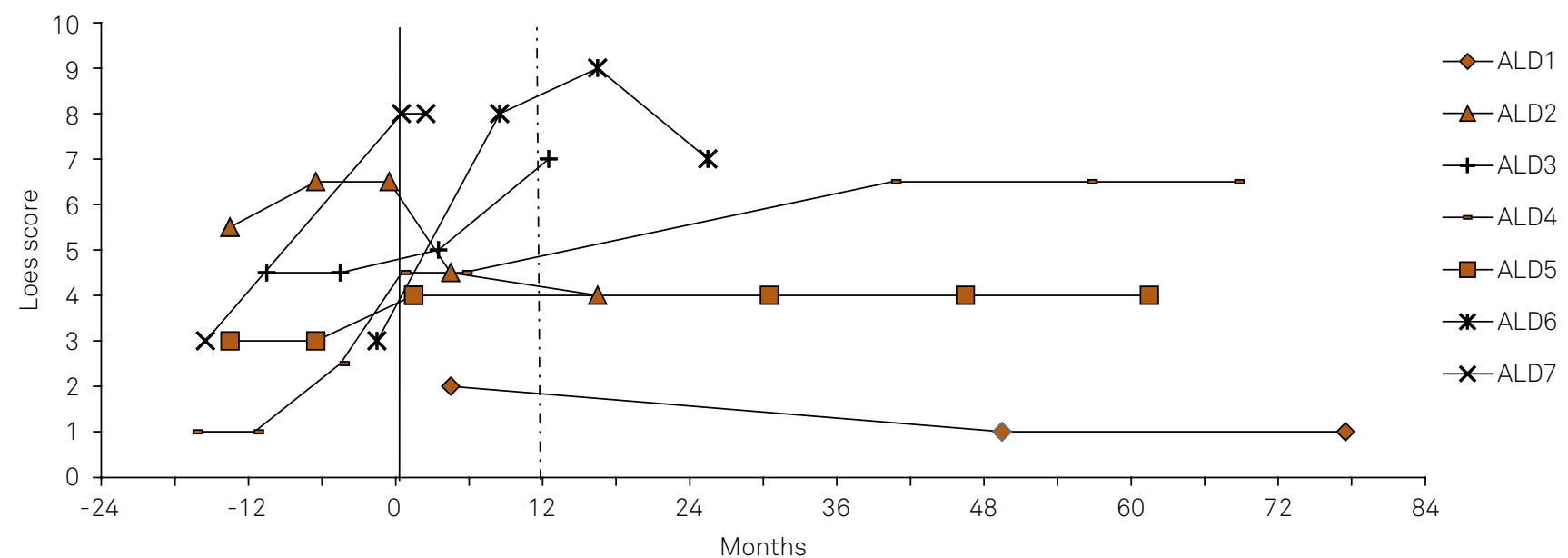

Figure 1. Loes score progression of seven CALD patients before and after HSCT. Individual dots indicate when brain magnetic resonance imaging was performed. The first MRI was the image obtained at the time of HSCT indication. Time zero (continuous line) indicates when HSCT was performed. The 12-month interval (dotted line) indicates the time window for efficacy.
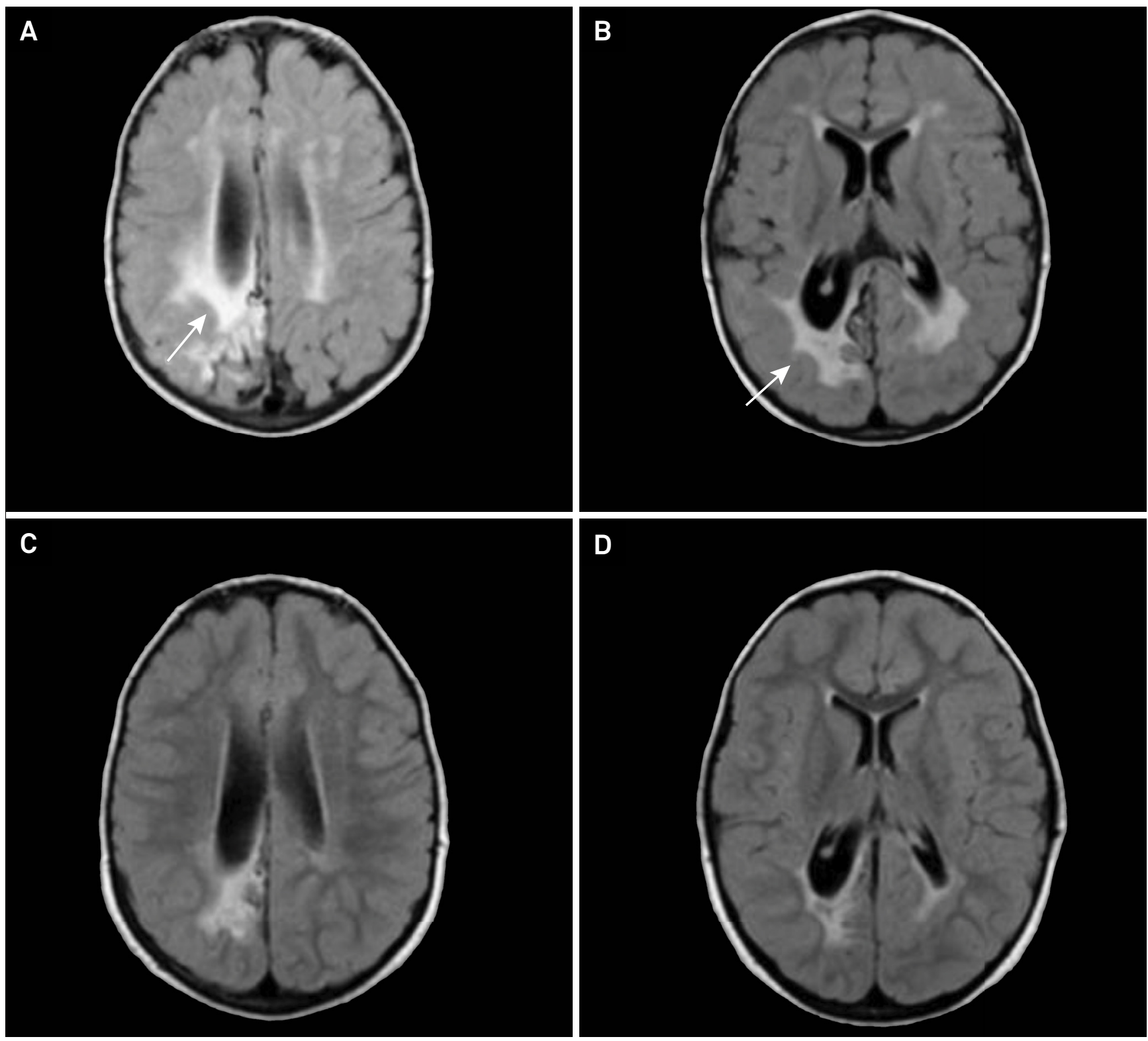

Figure 2. Brain FLAIR axial magnetic resonance images (MRI) of MPS-IH-10 patient. (A and B) MRI before HSCT. Hyperintensity lesions were mainly found in the periventricular and deep white matter (WM) (arrow). There was also mild ventricular enlargement. (C and D) MRI obtained three years after HSCT. Significant reduction of hyperintensities in the WM (arrow). The mild ventricular enlargement was smaller than before HSCT. 
HSCT, from opportunistic infection, with or without GVHD. Multiple reasons probably contributed to their deaths. In common, both deceased patients received grafts from unrelated donors, an already-known factor associated with increased risk of mortality ${ }^{26,27}$. Moreover, the CALD-7 patient started with symptoms in adulthood, another factor associated with higher HSCT morbidity and mortality risks ${ }^{27}$. Although mortality rates after HSCT in CALD of less than $5 \%$ have been reported ${ }^{7}$, none of the studies on HSCT outcomes published so far included adult forms ${ }^{15}$. A report on a 36-year-old CALD man showing cognitive deterioration in the previous year described the patient's death from GVHD three months after $\mathrm{HSCT}^{28}$.

The remaining nine individuals of the present report had sufficient follow-up time to be described. They were six CALD, two MLD and one MPS-IH patients.

One out of the six CALD patients surviving HSCT presented with several complications from HSCT and worsening of Loes score up to 12 months after HSCT. In contrast, the other five patients showed neurological stabilization after transplantation: CALD-1, CALD-2, CALD-4, CALD5 and CALD-6 (Table 2 and Figure 1). Mean age and Loes scores at the procedure were 10.9 years and 4 points. Mean Loes scores changed to 5.9 after a mean time of four years after their HSCTs; their neurological status was mostly stable. One adolescent patient (CALD-2) presented simultaneously with adrenomyeloneuropathy (AMN) and CALD at the time of HSCT. Nine years later, AMN findings worsened while CALD findings stabilized. All the other subjects showed normal functioning at school and in daily life activities. Signs of peripheral neuropathy appeared five and seven years after HSCT in two 16-year-old males (CALD-1 and CALD-4), probably related to AMN evolution. These results are in agreement with data showing that HSCT is effective in stabilizing CALD, but not $\mathrm{AMN}^{7,15}$.

Therapeutic results in MLD transplanted patients were completely divergent. While HSCT in patient MLD-8b seemed to be a success, it did not halt the disease progression in the adult form of patient MLD-9. Both patients shared the same genotype: the main difference was the clinical picture at the time of HSCT. Patient MLD-8b was a completely asymptomatic girl (Eichler 10) transplanted at nine years old, and her neurological examination remained normal until the last follow-up at 16 years of age. Although other juvenile onset patients remained stable after HSCT $^{29,30}$, the lack of association of genotype p.P426L/p.P426L with age at onset complicates the evaluation of HSCT success. For instance, two untreated patients homozygous for p.P426L were still asymptomatic at

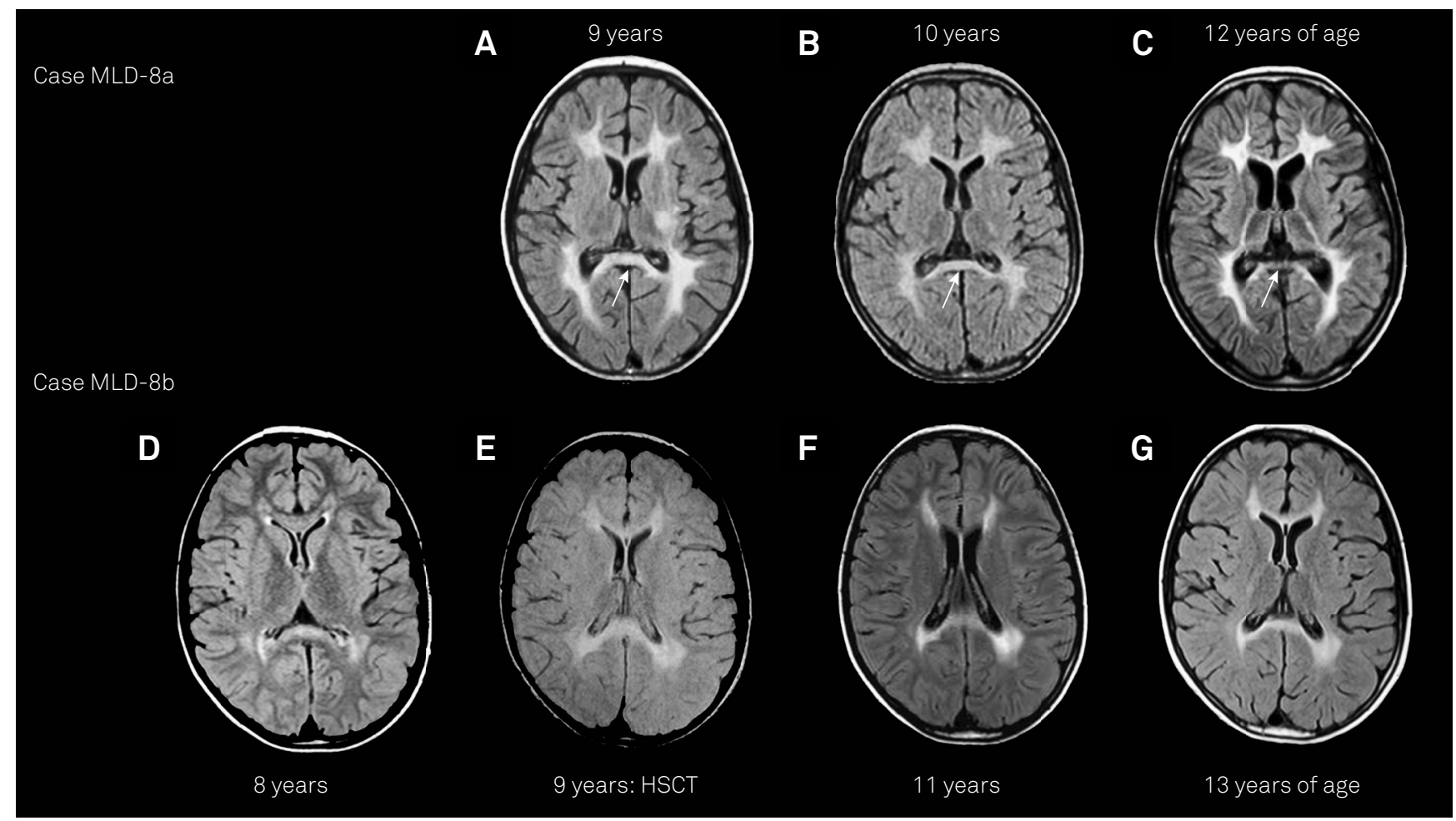

Figure 3. Brain FLAIR axial magnetic resonance images (MRI) of the transplanted patient MLD-8b and her non-transplanted brother MLD-8a. A, B and C. FLAIR axial MRIs from MLD-8a (non-tranplanted) patient. Images show hyperintense lesions diffusely distributed in the frontal, temporal, parietal and occipital white matter (WM) and in genu and splenium of the corpus callosum at $9(\mathrm{~A}), 10$ (B) and 12 years of age (C). There was a progressive worsening of brain atrophy during the follow up. White arrows highlight the progressive atrophy of the splenium of corpus callosum. D, E, F and G. FLAIR axial MRIs from MLD-8b patient show WM lesions distributed in the frontal, parietal and occipital lobes at 8 (D), 9 (E) (immediately before HSCT), 11 (F) and 13 years of age (G). Hyperintensities on the splenium of corpus callosum worsened until HSCT, with stabilization thereafter. The Eichler score was the same at $11(\mathrm{~F}), 13(\mathrm{G})$ and 14 years of age (image not shown). 
ages 14 and 322; our patient MLD-9 was also homozygous for p.P426L and had a disease onset at 19 years old. Late onset among p.P426L homozygotes points to longer follow-up observations to confirm the effectiveness of HSCT in these patients. It is important to note that evolution of patient MLD8a cannot be used as an untreated control for HSCT efficacy in patient MLD8b. His report emphasizes the importance of genetic counseling for the early, presymptomatic diagnosis of MLD. Patient MLD8a came to our attention too late, when an HSCT would not offer a favorable outcome. However, his diagnosis allowed the detection of patient MLD8b before disease onset. And indeed, two recent studies - a prospective cohort and case-control study - have raised evidence that HSCT is associated with a reasonable chance of disease stabilization if performed pre-symptomatically ${ }^{10,16}$.

Patient MLD-9 came for HSCT evaluation one year after the beginning of cognitive losses. An HSCT was offered to this patient based on the assumption that adult forms progress slower than early-onset forms and that there was a chance that microglial cross correction could happen before the development of more severe neurological deterioration. However, follow-up revealed that this was not the case. Soon after HSCT, he developed an akinetic mutism. Since therapeutic effects are expected some months (usually 12) after HSCT, MLD-9's deterioration was probably related to a rapid disease progression in an individual with alreadyextensive leukodystrophy on MRI at presentation. Recently, HSCT performed in four adult MLD patients was associated with stabilization four to 18 years after the procedure ${ }^{6}$. Three of them showed high Eichler scores, from 18 to 26, before transplantation. Patient series have reported either stabilization ${ }^{31}$ or failure after $\mathrm{HSCT}^{32}$ in juvenile and adult MLD patients. Better evidence favoring HSCT in juvenile MLD was recently obtained, when the neurological follow-up of 24 transplanted patients was better than those of 41 non-transplanted patients ${ }^{10}$. Several factors were associated with a better prognosis after HSCT: gross motor function preserved, IQ of at least 85, and age at onset older than four years.

I.

II.

III.

IV.

V.

Sulfatides = normal

p.P426L/c.1524+96A>G

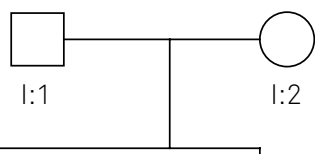

II.

V.
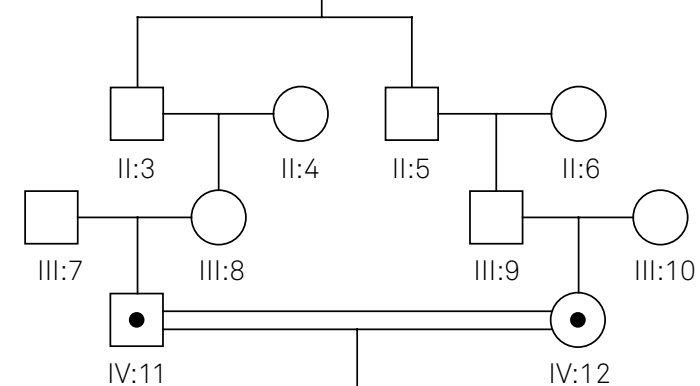

$\mathrm{ARSA}=11 \mathrm{nmol} / \mathrm{h} / \mathrm{mg}$ protein Sulfatides $=$ normal p.P426L/wt

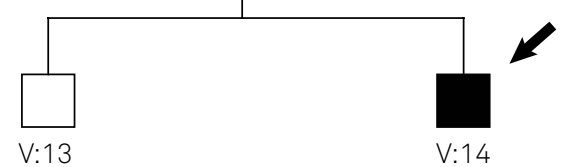

ARSA $=2.6 \mathrm{nmol} / \mathrm{h} / \mathrm{mg}$ protein Sulfatides $=$ normal c. $1524+96 \mathrm{~A}>\mathrm{G} / \mathrm{wt}$

ARSA $=$ undetectable Sulfatides $=$ increased p.P426L/p.P426L

Figure 4. Pedigree of MLD-9 family. Proband is indicated with a black arrow. Squares indicate males and circle, females; dark fill indicates affected individuals. Arylsulfatase A (ARSA) activity in leukocytes (normal range: $5-20 \mathrm{nmol} / \mathrm{hg} / \mathrm{mg}$ protein), urinary sulfatides, and ARSA genotypes are depicted for MLD-9 patient ( $V: 14$ individual), his parents IV:11 and IV:12, and his brother and bone marrow donor, V:13. Gene sequencing detected p.P426L as the pathogenic mutation in homozygosis in MLD-9 ( $\mathrm{V}: 14)$; both parents (IV:11 and IV:12) carried this mutation (were heterozygous), as expected. In addition, father (IV:11) and brother ( $V: 13)$ were carriers of the variant associated with ARSA pseudodeficiency, c.1524+96A>G.

Table 3. Factors potentially associated with unfavorable outcomes in the present patient series.

\begin{tabular}{|c|c|c|c|}
\hline \multirow[b]{2}{*}{ Factors } & \multirow[b]{2}{*}{ All } & \multirow[b]{2}{*}{ Favorable outcome } & \multirow{2}{*}{$\begin{array}{c}\text { Unfavorable outcome } \\
\begin{array}{c}\text { Death due to HSCT or worsening } \\
\text { of clinical picture }\end{array}\end{array}$} \\
\hline & & & \\
\hline & 11 & 7 & 4 (2 CALD, 1 MLD, 1 MPS-IH) \\
\hline HSCT related donor & 5 & 4 & 1 \\
\hline HSCT unrelated donor & 6 & 3 & 3 (two deaths) \\
\hline Adult-onset disease & 2 & 0 & 2 (one MLD and one CALD) \\
\hline $\begin{array}{l}\text { Loes score at the time of HSCT, in } \\
\text { CALD }\end{array}$ & 7 (2 to 8$)$ & 4 (2 to 6.5$)$ & 6.25 (4.5 and 8$)$ \\
\hline Mean (range) & & & \\
\hline $\begin{array}{l}\text { Disease duration before HSCT *, } \\
\text { in years }\end{array}$ & 0.94 (0 to 1.6$)$ & 0.8 (0 to 1.4$)$ & 1.2 (0.7 to 1.6$)$ \\
\hline Mean (range) & & & \\
\hline
\end{tabular}

*Disease duration = the difference between age at onset of cerebral signs and age at HSCT (see Table 2). HSCT: Hematopoietic stem cell transplantation; CALD: Cerebral from of X-linked adrenoleukodystrophy; MLD: Metechromatic Leukodystorphy; MPS-IH: mucopolysaccharidosis type I-Hurler. 
Early transplantation has been related to optimal long-term cognitive and language outcomes in MPS-IH ${ }^{9,14}$. Although our MPS-IH survivor (patient MPS-IH-10) was a late transplanted patient, her follow-up four years later was very encouraging. Clinical improvement, despite relatively low engraftment, should not be surprising. Studies in fibroblasts from MPS-I patients with different phenotypes and different genetic backgrounds confirmed that tiny differences in residual enzyme activity are related to important clinical differences $^{33}$. Several studies on HSCT in MPS-IH reported improvements in cardiopulmonary function, hearing and vision, and preservation of neurocognitive function ${ }^{9,14,34}$. Some of them documented that subsequent cognitive deterioration might occur after HSCT, mostly if it is performed late ${ }^{35}$. None of these studies, however, reported a reduction of brain lesions on MRI after HSCT, such as happened in this patient (Figure 2). Since this patient was not receiving enzyme replacement therapy, the clear reduction of periventricular and deep white matter lesions as well as the reduction in ventricular dilatation should be related to an HSCT effect.

Our cohort is small and included three different diseases. Even so, we looked for differences between patients with favorable and unfavorable outcomes after HSCT - considering death due to HSCT or deterioration of the clinical picture as the unfavorable outcomes (Table 3). In summary, better outcomes were obtained in patients whose HSCT was performed before adulthood, early in the disease clinical course, and from a related donor.

In conclusion, the present study showed short- to long-term results of neurological functions after HSCT in CALD, MLD and MPS-IH, in Brazil. Our survival rate was of $81 \%$ after a median of 3.7 years. While the results for the two adult forms were negative - one procedure-related death in a 28 -year-old CALD patient and a rapid deterioration in a 20 -year-old MLD patient - the response to HSCT was very satisfactory for seven of the other nine individuals. Better outcomes were obtained in those patients whose HSCT was performed early, as were the cases of CALD patients with a mean baseline Loes of 4 , and of the pre-symptomatic MLD individual. The outcome of the late-transplanted MPS-IH patient was also very satisfactory, being accompanied by reductions in the lesions seen in MRI.

\section{Acknowledgements}

We are grateful for all patients and families who participated in this study. We thank Dr. Greg Pastores and Prof. Edwin Kolodny for the molecular studies on patient MLD 8.

\section{References}

1. Krall WJ, Challita PM, Perlmutter LS, Skelton DC, Kohn DB. Cells expressing human glucocerebrosidase from a retroviral vector repopulate macrophages and central nervous system microglia after murine bone marrow transplantation. Blood. 1994;83(9):2737-48.

2. Rauschka H, Colsch B, Baumann N, Wevers R, Schmidbauer M, Krammer $\mathrm{M}$ et al. Late-onset metachromatic leukodystrophy: genotype strongly influences phenotype. Neurology. 2006;67(5):859-63. doi:10.1212/01.wnl.0000234129.97727.4d

3. Cable C, Finkel RS, Lehky TJ, Biassou NM, Wiggs EA, Bunin N et al. Unrelated umbilical cord blood transplant for juvenile metachromatic leukodystrophy: a 5-year follow-up in three affected siblings. Mol Genet Metab. 2011;102(2):207-9. doi:10.1016/j.ymgme.2010.10.002

4. Martin HR, Poe MD, Provenzale JM, Kurtzberg J, Mendizabal A, Escolar ML et al. Neurodevelopmental outcomes of umbilical cord blood transplantation in metachromatic leukodystrophy. Biol Blood Marrow Transplant. 2013;19(4):616-24. doi:10.1016/j.bbmt.2013.01.010

5. Solders M, Martin DA, Andersson, Remberger M, Andersson T, Ringdén 0 et al. Hematopoietic SCT: a useful treatment for late metachromatic leukodystrophy. Bone Marrow Transplant. 2014;:49(8):1046-51. doi:10.1038/bmt.2014.93

6. Beam D, Poe MD, Provenzale JM, Szabolcs P, Martin PL, Prasad V et al. Outcomes of unrelated umbilical cord blood transplantation for X-linked adrenoleukodystrophy. Biol Blood Marrow Transplant. 2007;13(6):665-74. doi:10.1016/j.bbmt.2007.01.082

7. Miller WP, Rothman SM, Nascene D, Kivisto T, DeFor TE, Ziegler RS et al. Outcomes after allogeneic hematopoietic cell transplantation for childhood cerebral adrenoleukodystrophy: the largest single-institution cohort report. Blood. 2011;118(7):1971-8 doi:10.1182/blood-2011-01-329235
8. Peters C, Shapiro EG, Anderson J, Henslee-Downey PJ, Klemperer MR, Cowan MJ et al. Hurler syndrome: Il. Outcome of HLA-genotypically identical sibling and HLA-haploidentical related donor bone marrow transplantation in fifty-four children. Blood. 1998;91(7):2601-8.

9. Ru MH, Boelens JJ, Das AM, Jones SA, van der Lee JH, Mahlaoui N et al. Enzyme replacement therapy and/or hematopoietic stem cell transplantation at diagnosis in patients with mucopolysaccharidosis type I: results of a European consensus procedure. Orphanet J Rare Dis. 2011;6(1):55. doi:10.1186/1750-1172-6-55

10. Groeschel S, Kühl JS, Bley AE, Kehrer C, Weschke B, Döring M et al. Long-term Outcome of Allogeneic Hematopoietic Stem Cell Transplantation in Patients With Juvenile Metachromatic Leukodystrophy Compared With Nontransplanted Control Patients. JAMA Neurol. 2016;73(9):1133-40. doi:10.1001/jamaneurol.2016.2067

11. Boelens JJ, Prasad VK, Tolar J, Wynn RF, Peters C. Current international perspectives on hematopoietic stem cell transplantation for inherited metabolic disorders. Pediatr Clin North Am. 2010;57(1):123-45. doi:10.1016/j.pcl.2009.11.004

12. Musolino PL, Lund TC, Pan J, Escolar ML, Paker AM, Duncan CN et al. Hematopoietic stem cell transplantation in the leukodystrophies: a systematic review of the literature. Neuropediatrics. 2014;45(3):169-74. doi:10.1055/s-0033-1364179

13. Aldenhoven M, Boelens JJ, Koning TJ. The clinical outcome of Hurler syndrome after stem cell transplantation. Biol Blood Marrow Transplant. 2008;14(5):485-98. doi:10.1016/j.bbmt.2008.01.009

14. Poe MD, Chagnon SL, Escolar ML. Early treatment is associated with improved cognition in Hurler syndrome. Ann Neurol. 2014;76(5):747-53. doi:10.1002/ana.24246 
15. Engelen M, Kemp S, Visser M, Geel BM, Wanders RJ, Aubourg $P$ et al. X-linked adrenoleukodystrophy (X-ALD): clinical presentation and guidelines for diagnosis, follow-up and management. Orphanet J Rare Dis. 2012;7(1):51. doi:10.1186/1750-1172-7-51

16. Boucher AA, Miller W, Shanley R, Ziegler R, Lund T, Raymond G. Long-term outcomes after allogeneic hematopoietic stem cell transplantation for metachromatic leukodystrophy: the largest single-institution cohort report. Orphanet J Rare Dis. 2015;10(1):94. doi:10.1186/s13023-015-0313-y

17. Jardim LB, Silva AC, Blank D, Villanueva MM, Renck L, Costa $M L$ et al. X-linked adrenoleukodystrophy: clinical course and minimal incidence in South Brazil. Brain Dev. 2010;32(3):180-90. doi:10.1016/j.braindev.2009.02.002

18. Moser HW, Moser AB. Measurement of saturated very long chain fatty acid in plasma. In: Hommes FA, editor. Techniques of diagnostic human biochemical genetics. Wiley, New York. 1991, pp. 177-191.

19. Virgens MY, Siebert M, Bock H, Burin M, Giugliani R, Saraiva-Pereira ML. Genotypic characterization of Brazilian patients with infantile and juvenile forms of metachromatic leukodystrophy. Gene. 2015;568(1):69-75. doi:10.1016/j.gene.2015.05.016

20. Loes DJ, Hite S, Moser H, Stillman AE, Shapiro E, Lockman L et al. Adrenoleukodystrophy: a scoring method for brain MR observations. AJNR Am J Neuroradiol. 1994;15(9):1761-6.

21. Eichler F, Grodd W, Grant E, Sessa M, Biffi A, Bley A et al. Metachromatic leukodystrophy: a scoring system for brain MR imaging observations. AJNR Am J Neuroradiol. 2009;30(10):1893-7. doi:10.3174/ajnr.A1739

22. Tutschka PJ, Copelan EA, Klein JP: Bone marrow transplantation for leukemia following a new busulfan and cyclophosphamide regimen. Blood. 1987;70(5):1382-8.

23. Cartier N, Aubourg P. Hematopoietic stem cell gene therapy in Hurler syndrome, globoid cell leukodystrophy, metachromatic leukodystrophy and X-adrenoleukodystrophy. Curr Opin Mol Ther. 2008;10(5):471-8. doi:

24. Jardim LB, Villanueva MM, Souza CF, Netto CB. Clinical aspects of neuropathic lysosomal storage disorders. J Inherit Metab Dis. 2010;33(4):315-29. doi:10.1007/s10545-010-9079-5

25. Lange MC, Teive HAG, Troiano AR, Bitencourt M, Funke VA, Setúbal DC et al. Bone marrow transplantation in patients with storage diseases: a developing country experience. Arq Neuropsiquiatr. 2006;64(1):1-4. doi:10.1590/S0004-282X2006000100001

26. Peters C, Charnas LR, Tan Y, Ziegler RS, Shapiro EG, DeFor T et al. Cerebral $X$-linked adrenoleukodystrophy: the international hematopoietic cell transplantation experience from 1982 to 1999. Blood. 2004;104(3):881-8. doi:10.1182/blood-2003-10-3402

27. Boelens JJ, Aldenhoven M, Purtill D, Ruggeri A, Defor T, Wynn R et al. Outcomes of transplantation using various hematopoietic cell sources in children with Hurler syndrome after myeloablative conditioning. Blood. 2013;121(19):3981-7. doi:10.1182/blood-2012-09-455238

28. Fitzpatrick AS, Loughrey CM, Johnston P, McKee S, Spence W, Flynn et al. Haematopoietic stem-cell transplant for adult cerebral adrenoleukodystrophy. Eur J Neurol. 2008;15(3):e21-2. doi:10.1111/j.1468-1331.2007.02048.x

29. Ding X-Q, Bley A, Kohlschütter A, Fiehler J, Lanfermann H. Long-term neuroimaging follow-up on an asymptomatic juvenile metachromatic leukodystrophy patient after hematopoietic stem cell transplantation: evidence of myelin recovery and ongoing brain maturation. Am J Med Genet A. 2012;158A(1):257-60. doi:10.1002/ajmg.a.34389

30. Krägeloh-Mann I, Groeschel S, Kehrer C, Opherk K, Nägele T, Handgretinger R et al. Juvenile metachromatic leukodystrophy 10 years post transplant compared with a non-transplanted cohort. Bone Marrow Transplant. 2013;48(3):369-75. doi:10.1038/bmt.2012.155

31. Egmond ME, Pouwels PJW, Boelens JJ, Lindemans CA, Barkhof F, Steenwijk $\mathrm{MD}$ et al. Improvement of white matter changes on neuroimaging modalities after stem cell transplant in metachromatic leukodystrophy. JAMA Neurol. 2013;70(6):779-82. doi:10.1001/jamaneurol.2013.629

32. Smith NJ, Marcus RE, Sahakian BJ, Kapur N, Cox TM. Haematopoietic stem cell transplantation does not retard disease progression in the psycho-cognitive variant of late-onset metachromatic leukodystrophy. J Inherit Metab Dis. 2010;33(s3):S471-5. doi:10.1007/s10545-010-9240-1

33. Oussoren E, Keulemans J, Diggelen OP, Oemardien LF, Timmermans RG, Ploeg AT et al.. Residual $\alpha$-L-iduronidase activity in fibroblasts of mild to severe Mucopolysaccharidosis type I patients. Mol Genet Metab. 2013;109(4):377-81. doi:10.1016/j.ymgme.2013.05.016

34. Prasad VK, Kurtzberg J. Transplant outcomes in mucopolysaccharidoses. Semin Hematol. 2010;47(1):59-69. doi:10.1053/j.seminhematol.2009.10.008

35. Shapiro E, Guler OE, Rudser K, Delaney K, Bjoraker K, Whitley $C$ et al. An exploratory study of brain function and structure in mucopolysaccharidosis type I: long term observations following hematopoietic cell transplantation (HCT). Mol Genet Metab. 2012;107(1-2):116-21. doi:10.1016/j.ymgme.2012.07.016 


\section{CALD Patients}

\section{Patient CALD 1, Family A}

CALD1, male (originally reported as patient $\mathrm{G}$ in Lange et al. ${ }^{25}$ ), presented with Addison disease at seven years of age. X-ALD diagnosis was made by very long-chain fatty acid (VLCFA) analysis at eight years old and he was the index patient of his family. The first magnetic resonance imaging (MRI) was normal; the second exam performed at eight years, 10 months disclosed white matter (WM) lesions in the internal capsules and anterior portions of the midbrain (Loes score of 2). The neurological examination and IQ evaluation were both normal (Table 2). Molecular analysis detected a p.L628Q mutation at $A B C D 1$ of CALD1 and his mother. His five-year-old sister had a normal VLCFA profile, and was the only HLA identical relative detected in the family. The staff team, parents and the girl agreed, and she was the donor for the HSCT carried out when CALD1 was nine years, five months. He was discharged 40 days after an almost uneventful period at the hospital. The last follow-up was done at 16 years of age: CALD1 was performing normally at school and presented with no complaints. At neurological examination, there was a strength grade 4/5 in both anterior tibial muscles; hammer toes, absent plantar responses; and reduced vibratory perception (lasting 11 and 9 seconds on toes). All other findings were normal. An MRI performed after HSCT showed a reduction of the lesions in the brainstem (Loes score of 1; Figure 1). Biochemical markers were unchanged (Table 2).

\section{Patient CALD 2, Family B}

CALD2, male, 18 years old, came for evaluation as the only brother of a previous CALD patient. CALD2 presented with loss of strength in lower limbs in the previous four months. Brisk reflexes and a peripheral axonal neuropathy on neurophysiology pointed to an adrenomyeloneuropathy (AMN) phenotype. The VLCFA analysis confirmed X-ALD; ACTH levels were normal. The p.A232fsX64 mutation was detected in $A B C D 1$. His brain MRIs disclosed lesions at the splenium of corpus callosum; in periventricular and central WM of parieto-occipital and right frontal lobes; and in the internal capsules. Loes scores evolved from 5.5 to 6.5 in a seven-month interval. A paternal uncle was HLA identical to CALD2 and was his bone marrow donor. CALD2 was discharged 33 days after HSCT, and returned only once, fourteen months after transplantation. Neurological examination had not changed, but follow-up MRIs showed improvements (Loes score of 4.5 and 4, after four and 16 months post-HSCT) (Table 2 and Figure 1). CALD2 and his father were very frustrated because HSCT did not improve the impairments due to AMN, and never came back to follow-up visits. The last contact was in 2013 by phone: CALD2 was 25 years old and wanted to review the genetic risks to his offspring.

\section{Patient CALD 3, Family B}

A cousin of the former patient CALD2, CALD3, male, was found to carry the same mutation - p.A232fsX64 - at ABCD1. Followed with periodic brain MRIs since five years old, he was diagnosed as the third CALD in the family. At six years, six months, CALD3 presented with ankle clonus and attention deficit to auditory stimuli, plus hyperactivity. His former MRIs were normal. After a transient loss of follow-up, a new MRI was obtained at eight years old and showed bilateral WM lesions in the medial geniculate bodies, lateral lemniscus, brachium of inferior colliculus and in the projection fibers of the brainstem; and of the left internal capsule (Loes score of 4.5). An HSCT from an unrelated donor was performed in September 2014. Two months later, CALD3 presented with a hemorrhagic cystitis and in the following month, GVHD with gastrointestinal and skin manifestation, refractory to treatment. Brain MRI, three months after HSCT, showed a mild worsening of lesions (Loes score of 5). In April 2015, he was still in hospital, treating recurrent infections (urinary and intestinal). One year after HSCT, CALD3 presented with a marked worsening, with mutism, spasticity and tetraparesis; he was discharged with a Loes score of 7.

\section{Patient CALD 4, Family C}

CALD4, male (reported as patient $\mathrm{H}$ in Lange et $\mathrm{al}^{25}$ ), belongs to a large X-ALD family where mutation p.R518Q segregates. Followed as an asymptomatic carrier with normal MRIs since five years of age, CALD4 turned into an Addison-only phenotype when he was seven years old. The first WM lesions appeared when he was nine years, six months, in the right periventricular and central parieto-occipital lobe WM (Loes score of 1), when CALD was diagnosed. An unrelated donor was found 16 months later: by this time, the Loes score had progressed to 4.5 points and neurological examination disclosed brisk patellar reflexes, only. He underwent HSCT at 10 years, 10 months. CALD4 was discharged at day 36 after HSCT and no complications were reported. Clonus, brisk reflexes in lower limbs, and a Babinski sign were observed at 14 years of age. At 16 years old, the neurological examination returned to normal; an ENMG disclosed a mixed axonal and demyelinating peripheral neuropathy. The MRI lesions were stabilized (Loes score of 6.5) during this five-year period after the bone marrow transplant (BMT) (Table 2 and Figure 1).

\section{Patient CALD 5, Family D}

CALD5, male (reported as patient I in Lange et al. ${ }^{25}$ ), was admitted to our hospital at seven years, six months to investigate adrenal insufficiency. Very long-chain fatty acid analysis confirmed the diagnosis of X-ALD. The neurological examination was normal, whereas the MRI disclosed WM lesions in the splenium of corpus callosum and in the 
periventricular and central WM of the parieto-occipital lobes (Loes score of 3). Molecular studies detected the p.S358X mutation of the $A B C D 1$ gene of CALD5 and in his mother; no other relative carried this mutation. An umbilical cord HSCT was undertaken when he was eight years, five months. The patient was discharged 42 days after HSCT, with no complications. Five years after the BMT, the neurological examination was normal and the MRI lesions were stable (Loes score of 5) (Table 2 and Figure 1).

\section{Patient CALD 6, Family E}

CALD6, male, was a brother of an index-patient with an advanced form of CALD (Loes score of 13). At the time of the index-patient diagnosis, CALD6 was two years old. His VLCFA profile was characteristic of X-ALD, and his molecular studies showed that he carried the mutation found in his family - p.A401W. His MRIs were normal up to five years old. On follow-up at six years old, brisk reflexes were found on his left side. The MRI at six years, three months showed lesions in the genu of corpus callosum, and in central and periventricular WM of the frontal lobes (Loes score of 3 ). An HSCT was carried out when he was six years, six months old: an older sister, HLA identical and a non-carrier of the p.A401W mutation, was the donor. CALD6 was discharged 50 days after HSCT, with no complications. A new MRI performed eight months later showed new lesions in the splenium of corpus callosum, internal capsules subcortical frontal lobes and in projection fibers of the brainstem (Loes score of 8). Sixteen months after the BMT, at eight years old, a third MRI gave a Loes score of 9; brisk reflexes on his left side were still present, plus a complaint of memory difficulties. Two years after BMT, at eight years, eight months (July, 2014), a forth MRI showed a reduction of the WM lesions and a Loes score of 7 . Memory difficulties were no longer present (Table 2 and Figure 1).

\section{Patient CALD 7, Family F}

CALD7, male, a successful systems analyst, presented with loss of coordination and strength in his right arm when he was 27 years old. Initially diagnosed as an ischemic event, the clinical picture progressed to severe hemiparesis in the following months. Mental functioning was normal. The MRI showed lesions in periventricular and central WM of the left temporal lobe, and bilateral periventricular and deep WM lesions in the parieto-occipital lobes, body of corpus callosum, left basal ganglia, left internal capsule, left Meyer's loop, left projection fibers of the brainstem, and global atrophy (Loes score of 8). The VLCFA analysis diagnosed X-ALD; he and several relatives carried the p.P560L mutation in $A B C D 1$. An unrelated, mismatched donor was found nine months after the diagnosis of CALD. Immediately before HSCT, the patient presented with hemiparesis, dysarthria and a conduction aphasia, without any further cognitive deterioration. The Loes score was the same in a new MRI. After HSCT, several complications followed, including seizures, chronic GVHD and hemorrhagic cystitis. Cognitive functions were maintained. The patient died nine months after HSCT from an opportunistic infection (Table 2 and Figure 1).

\section{MPS IH Patients}

\section{Patient MPS-IH10, Family I}

MPS-IH10, female, was referred for genetic evaluation at eight months of age due to recurrent upper air infections, hepatomegaly, failure to thrive, developmental delay and coarse facies. MPS-IH10 was abandoned by her parents at birth and lived in an orphanage. At physical examination, weight and length were under percentile 3 and the cephalic perimeter under percentile 10. There was mild facial coarsening, enlarged tongue, thoracolumbar gibbus, hepatomegaly and moderate development delay (steady head, good interaction, social laughter, did not sit without support). A CT scan showed generalized cortical atrophy. The investigation disclosed high levels of urinary glycosaminoglycans (GAGs) - $601 \mathrm{ug} / \mathrm{mg}$ creat (normal range: 133-274) - and low activity of $\alpha$-L-iduronidase (IDUA) in leukocytes $-0.17 \mathrm{nmol} / \mathrm{h} / \mathrm{mg}$ protein (normal range: $32-56$ ), both compatible with the diagnosis of MPS I. Sequencing of IDUA revealed that MPS-IH10 was homozygous for $\mathrm{p}$.W402X, a mutation previously related to the Hurler type of MPS I or

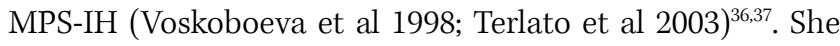
started to be evaluated for HSCT. At 16 months of age, enzyme replacement therapy with laronidase was started with improvement in her clinical status and respiratory condition. At 24 months of age, brain MRI showed T2/FLAIR hyperintensities, mainly in the periventricular and deep WM associated with mild ventricular enlargement (Figure 4A and B). By that time, auditory evoked potentials showed bilateral hearing loss. An unrelated donor was found and HSCT was performed at 27 months of age. MPS-IH10 was discharged 195 days after HSCT. She presented with acute GVHD with skin and gastric involvement. Enzyme replacement therapy was discontinued after HSCT. Four years after the HSCT, MPSIH10 was in quite good general condition, very active, walking without support. Communication was nonverbal, though enough for her daily needs. The typical face, weight and height on percentile 3 , gibbosity, and joint restriction in the hands, arms and legs were still present. There was no hepatomegaly on ultrasound. Echocardiogram showed thickening of the leaflets of the aortic and mitral valves without changes in transvalvular flow. Diameters of interventricular and atrial septum were normal. Although outside the normal range, she has shown progressive improvement of motor skills, language and expression. Since HSCT, she has not shown any cognitive loss. No significant skeletal improvement was observed in this four-year interval. A clear improvement in the brain lesions appeared on MRI performed three years after HSCT, with significant reduction of periventricular and deep 
WM lesions. The mild ventricular enlargement was also reduced (Table 2 and Figure 4C and D).

\section{Patient MPS-IH11, Family J}

MPS-IH11, male, was referred for genetic evaluation at 11 months of age due to recurrent upper air infections and developmental delay. Urinary GAGs of $481 \mathrm{ug} / \mathrm{mg}$ creat and IDUA activity of $0.18 \mathrm{nmol} / \mathrm{h} / \mathrm{mg}$ protein in leukocytes established the diagnosis of MPS I. Molecular analysis disclosed a p.W402X homozygous genotype of IDUA, classically associated with MPS-IH. MPS-IH11 was the first child from consanguineous parents. On examination, a clear-cut MPS phenotype was present, with gingival hypertrophy, impacted teeth, restriction of the extension of fingers, large umbilical hernia, and hepatomegaly. Enzyme replacement therapy with laronidase was started at 17 months of age; by this time, he could stand without support, but was unable to walk or to speak. Brain atrophy, ventriculomegaly and mega cisterna magna were found on MRI. Thickening of the periodontal tissues determined spinal cord stenosis at C1-C2 level. The first HSCT was done at 22 months of age. Chimerism was progressively lost, with 73, 58 and $10 \%$ after one, two and eight months. The IDUA activities also fell, with 40, 42 and $12 \mathrm{nmol} / \mathrm{h} / \mathrm{mg}$ protein after one, two and five months, respectively. Due to progressive loss of the donor cell, a second transplant with the same donor was performed, at two years nine months. MPS-IH11 died 30 days later due to viral infection and respiratory complications (Table 2). 